\title{
Lipids, Anthropometric Measures, Smoking and Physical Activity Mediate the Causal Pathway from Education to Breast Cancer in Women: A Mendelian Randomization Study
}

\section{Hongkai Li}

Shandong University Cheeloo College of Medicine

\section{Lei Hou}

Shandong University Cheeloo College of Medicine

\section{Yuanyuan $\mathrm{Yu}$}

Shandong University Cheeloo College of Medicine

\section{Xiaoru Sun}

Shandong University Cheeloo College of Medicine

Xinhui Liu

Shandong University Cheeloo College of Medicine

\section{Ran Yan}

Shandong University Cheeloo College of Medicine

Yifan Yu

Shandong University Cheeloo College of Medicine

\section{Sijia Wu}

Shandong University Cheeloo College of Medicine

Yina He

Shandong University Cheeloo College of Medicine

\section{Yutong Wu}

Shandong University Cheeloo College of Medicine

\section{Li He}

Shandong University Cheeloo College of Medicine

\section{Fuzhong Xue ( $\nabla$ xuefzh@sdu.edu.cn )}

School of Public Health of Shandong University, Health Big Data Institute of Shandong University https://orcid.org/0000-0003-0378-7956

\section{Research article}

Keywords: education, estrogen receptor, Mendelian randomization, mediators, meta-analysis 
Posted Date: April 20th, 2021

DOI: https://doi.org/10.21203/rs.3.rs-418843/v1

License: (c) (1) This work is licensed under a Creative Commons Attribution 4.0 International License. Read Full License

Version of Record: A version of this preprint was published at Journal of Breast Cancer on January 1st, 2021. See the published version at https://doi.org/10.4048/jbc.2021.24.e53. 


\section{Abstract \\ Background}

Our objective is to investigate whether obtaining a higher level of education was causally associated with lower breast cancer risk and to identify the causal mechanism linking them.

\section{Methods}

Firstly, we performed a meta-analysis for educational attainment on breast cancer using 33 MR studies, including 15 case-control studies, 10 cross-sectional studies, and 8 cohort studies. Secondly, the main data analysis used publicly available summary-level data from two large GWAS consortia (Breast Cancer Association Consortium [BCAC] and the Social Science Genetic Association Consortium [SSGAC]). Mendelian randomization (MR) analysis used 65 genetic variants derived from the SSGAC as instrumental variables for years of schooling. The outcomes were the overall breast cancer risk $(122,977$ cases/105,974 controls in women) and its two subtypes: estrogen receptor (ER)-positive breast cancer (ER+: 69,501 cases) and ER-negative breast cancer (ER-: 21,468 cases). Furthermore, six additional consortia were analyzed to investigate the causal pathways from education to breast cancer. The fixed and random effects inverse variance weighted methods were used to estimate the causal effects, along with other additional MR methods as sensitivity analyses.

\section{Results}

The results showed that each additional standard deviation of 4.2 years of education was causally associated with a $27 \%$ lower risk of ER- $(\mathrm{OR} 0.73,95 \% \mathrm{Cl}[0.64,0.84]$; P-value < 0.001$)$. However, very weak causal relationship was found with overall breast cancer and no causal association with ER + risk, consistent with the sensitivity analyses. A genetic predisposition for higher education was causally associated with lower ER- risk and was found to be partially related to hip circumference, body mass index, triglyceride and HDL levels, smoking, and physical activity.

\section{Conclusion}

A low level of education is a causal risk factor in the development of ER- as it is associated with a poor lipid profile, anthropometric measurements, smoking, and types of physical activity.

\section{Background}

Breast cancer is the second leading cause of mortality among European women and almost 1 in 8 women develop breast cancer during their lifetime[1-2]. Each year, approximately 17 million new cases have been reported[3-4]. With the increasing burden of breast cancer, it is imperative to identify modifiable risk 
factors for prevention. Education is a key component of socioeconomic status (SES) and may lower breast cancer risk by altering the lipids profile, anthropometric measurements, physical activity, smoking, and so on [25]. Numerous observational studies have investigated the relationship between education and breast cancer, but have widely inconsistent results[5-9]. For instance, a case-control study and a cohort study suggested opposite outcomes for the relationship between education level and breast cancer[1011]. The former found an inverse association of educational level with breast cancer risk $(O R=0.17)$, whereas the latter showed that in contrast with women who completed less than 9 years of education, university graduates had a higher probability to be diagnosed with in situ $H R=1.44$ and invasive $H R=1.28$ breast cancer. These contradictory results may be attributed to the limitations of traditional observational studies including unmeasured confounding and reverse causation.

The instrumental variable (IV) method exploits a natural experiment to obtain a causal association of an exposure on an outcome. A valid instrument must satisfy the following three assumptions: (1) Relevance - IV $(G)$ is robustly related to exposure $(X)$; (2) Exchangeability - IV $(G)$ is independent of any unobserved confounders $(U)$ of the exposure and outcome relationship; (3) Exclusion restriction - IV $(G)$ affects the outcome $(Y)$ only through the exposure $(X)$ [12]. In observational data, employing genetic variants as instrumental variables has been termed "Mendelian randomization (MR)." MR analyses using summarized data have recently become popular due to a large number of published genome-wide association studies (GWAS) publicly available in large sample populations, [13] significantly increasing its statistical power.

Using the MR approach, several reports have found that the level of education is causally associated with myopia [15], lung cancer [14] and coronary heart disease [13]. However, to the best of our knowledge, this is the first report on the relationship between educational attainment and breast cancer. In this study, we used two-sample MR analyses to identify the potential causation between education level and breast cancer and its ER subtypes. Furthermore, we also investigated the causal pathways linking them.

\section{Methods And Materials}

\section{Genetic variants related to educational attainment}

Educational attainment (EA) is measured as the number of years of schooling completed. A large genetic association study reported by Lee et al. [43] identified 317 single nucleotide polymorphisms (SNPs) robustly associated with educational attainment in the Social Science Genetic Association Consortium (SSGAC) at a GWAS threshold of statistical significance [766, 345 participants of European descent; Pvalue $<5 \times 10^{-8}$; linkage disequilibrium (LD) $r^{2}<0.001$ ] (Table 1). These 317 SNPs explain $2.03 \%$ of the variation in educational attainment across individuals. The F statistic is larger than the "rule of thumb" of 10 [12], which means the instruments used strongly predict educational attainment. Thus, it is sufficient to generate a strong genetic instrument based on these 317 SNPs. Therefore, in this study, we used only 317 SNPs and summary data collected from the SSGAC. 
Table 1

Details of studies included in Mendelian randomization analyses

\begin{tabular}{|c|c|c|c|c|}
\hline Consortium & Phenotype & Participant & year & Web source \\
\hline SSGAC & $\begin{array}{l}\text { Years of } \\
\text { schooling }\end{array}$ & 766345 & 2018 & www.thessgac.org/data \\
\hline BCAC & Breast cancer & 228951 & 2017 & bcac.ccge.medschl.cam.ac.uk/ \\
\hline GLGC & Lipids & 188557 & 2013 & csg.sph.umich.edu/willer/public/lipids2013/ \\
\hline GIANT & $\begin{array}{l}\text { Anthropometric } \\
\text { measures }\end{array}$ & 224459 & 2015 & portals.broadinstitute.org/collaboration/giant \\
\hline UK Biobank & $\mathrm{BMI}$ & 461460 & 2018 & www.ukbiobank.ac.uk/ \\
\hline UK Biobank & Sleep duration & 128266 & 2018 & www.ukbiobank.ac.uk/ \\
\hline MRC-IEU & $\begin{array}{l}\text { Physical } \\
\text { activities }\end{array}$ & 160376 & 2018 & gwas.mrcieu.ac.uk/ \\
\hline TAG & Smoke & 74053 & 2010 & $\begin{array}{l}\text { www.med.unc.edu/pgc/results-and- } \\
\text { downloads }\end{array}$ \\
\hline \multicolumn{5}{|c|}{$\begin{array}{l}\text { SSGAC, Social Science Genetic Association Consortium; GLGC, Global Lipids Genetics Consortium; } \\
\text { BCAC, Breast Cancer Association Consortium; MAGIC, Meta-Analyses of Glucose and Insulin-related } \\
\text { traits Consortium; MAGIC, Meta-Analyses of Glucose and Insulin-related traits Consortium; MRC-IEU, } \\
\text { MRC Integrative Epidemiology Unit; TAG, Tobacco and Genetics consortium. }\end{array}$} \\
\hline
\end{tabular}

\section{GWAS summary level data on breast cancer}

We retrieved GWAS summary data on breast cancer for European descent from the Breast Cancer Association Consortium (BCAC) [23] (Table 1). Results were available for 291 of the 317 EA-associated leading SNPs for breast cancer subtypes (Supplementary Table 1): overall breast cancer $(122,977$ cases/105,974 controls), estrogen receptor (ER)-positive breast cancer (69,501 cases/105,974 controls), and ER-negative breast cancer (21,468 cases/105,974 controls). Ten SNPs (rs12134151, rs13130765, rs1455350, rs2414072, rs2478208, rs2545798, rs320693, rs60483752, rs6867851 and rs7920624) were removed from the analysis for being palindromic with intermediate allele frequencies. We used summary data from four databases: (1) OncoArray Consortium (61,282 cases and 45,494 controls); (2) Collaborative Oncological Gene-environment Study (iCOGS: 46,785 cases and 42,892 controls); (3) 11 other breast cancer genome-wide association studies (GWAS; 14,910 cases and 17,588 controls); (4) Combination of the above three databases.

\section{Other breast cancer-risk factor data}

Summary results from genome-wide association meta-analyses for lipids were obtained from four genetic consortia, including HDL- and LDL- and total cholesterol, triglycerides, anthropometric measurements (hip and waist circumference, waist-to-hip ratio, height and body mass index (BMI)), smoking, sleep duration, and physical activities [18]. The websites used for data collection and consortia are listed in Table 1. All summary statistics obtained from analyses are limited to European descent. The statistical analyses 
included linear/logistic regression coefficients (beta/log[OR]), standard errors, and P-values for genetic association analysis.

\section{Statistical analyses}

First, we carried out a meta-analysis of all published observational studies exploring the relationship of educational attainment levels on breast cancer. We searched in PubMed, Medline, Embase, and Web of Science for studies containing the term 'education' or 'schooling' and 'breast cancer' up to 21 October 2020. We excluded publications that (i) were conference abstracts, letters, commentaries, editorials, reviews, study proposals, or theoretical papers; (ii) the main exposure is not education; (iii) education as an outcome. Then, we pooled the study-specific estimates by using a random-effects model for metaanalysis. We evaluated the study heterogeneity by calculating the $I^{2}$ statistic from Cochran's $Q$ test.

Multiple MR approaches were used to obtain estimates of educational attainment on breast cancer and its ER subtypes. We conducted fixed and random effects inverse variance weighted (IVW) meta-analysis [12, 44] of the Wald ratio for individual SNPs. We detected heterogeneity to determine whether to use fixed or random effects IVW. The IVW method assumes that all SNPs are valid instruments, which satisfies the MR assumptions. Three additional MR methods were also used as sensitivity analyses to assess the robustness of the results: MR-Egger regression and the weighted median and the weighted mode methods. The intercept of the MR-Egger regression provides an estimate of the average pleiotropic effect over all SNPs. If it differs from zero, it indicates the presence of horizontal pleiotropy. We also performed a leave-one-out analysis in which we sequentially omitted one SNP at a time to determine whether the MR estimate was driven or biased by a single SNP.

To investigate potential mechanisms from education to breast cancer, we applied network MR to explore potential mediators on this causal pathway. We selected the potential mediators based on existing literature, including lipids (HDL-, LDL-, and total cholesterol, and triglycerides), anthropometric measures (waist and hip circumference, waist-to-hip ratio, height, and BMI), smoking, sleep duration, and physical activities, which can be risk factors of breast cancer. We first performed MR to estimate the causal effects of educational attainment on these risk factors. Then, the additional MR analyses were performed for risk factors on breast cancer if EA is shown to have a causal effect on the above risk factors.

The results were interpreted via odds ratios (OR), 95\% confidence intervals (CI), and $P$-value, which provided an estimate of relative risk caused by each standard deviation (SD) (3.6 years) increase in the years of schooling. Based on a simulation study [24] on sample overlap and the degree of bias in MR analysis, a less than $5 \%$ degree of overlap is not considered significant. The proportion of sample overlap in the summary data we used in MR analyses is within the acceptable range and does not lead to an estimation bias. The R package TwoSampleMR (v0.5.1) was used for all the above MR methods (version 3.6.3). The calculation on MR analysis can be found at http://cnsgenomics.com/shiny/mRnd/.

\section{Results}




\section{Meta-analysis for educational attainment on breast cancer}

After applying our inclusion and exclusion criteria, $33 \mathrm{MR}$ studies (Additional table) were included in our meta-analysis. Forest plots of the meta-analysis can be found in Supplementary Fig. 23-25. These studies include 15 case-control studies, 10 cross-sectional studies, and 8 cohort studies. Significant heterogeneity between these studies was found when calculating the $\mathrm{I}^{2}$ value from Cochran's $Q$ test. Then we performed a random-effects model for meta-analysis. The meta-analysis results from the three studies revealed a positive association between educational attainment and breast cancer.

\section{Causal effect from education to breast cancer}

A large heterogeneity was found in several databases, thus we performed random-effects IVW. For the databases with no heterogeneity, we performed fixed effects IVW. Results for the heterogeneity test are listed in Table 2. There was no directional pleiotropy in all analysis. 
Table 2

Heterogeneity test and MR-Egger pleiotropy test of the causal effects of education attainment on risk of breast cancer and its subtypes

\begin{tabular}{|c|c|c|c|c|c|c|}
\hline \multirow[t]{3}{*}{ Outcome } & \multicolumn{4}{|c|}{ Heterogeneity test } & \multirow{2}{*}{\multicolumn{2}{|c|}{$\begin{array}{l}\text { Pleiotropy test } \\
\text { MR-Egger }\end{array}$}} \\
\hline & \multicolumn{2}{|l|}{ IVW } & \multicolumn{2}{|l|}{ MR-Egger } & & \\
\hline & $\mathbf{Q}$ & Pval & $\mathbf{Q}$ & Pval & intercept & Pval \\
\hline \multicolumn{7}{|l|}{ Breast cancer } \\
\hline Combination & 622.660 & $<0.001^{\star}$ & 620.082 & $<0.001^{\star}$ & 0.003 & 0.274 \\
\hline Oncoarray & 409.585 & $<0.001^{\star}$ & 405.472 & $0.001^{*}$ & 0.005 & 0.088 \\
\hline iCOGS & 423.556 & $<0.001^{*}$ & 423.517 & $0.010 *$ & -0.001 & 0.870 \\
\hline GWAS & 376.679 & $<0.001^{\star}$ & 375.888 & 0.809 & 0.004 & 0.436 \\
\hline \multicolumn{7}{|l|}{ ER+ } \\
\hline Combination & 525.272 & $<0.001^{\star}$ & 523.770 & $<0.001^{\star}$ & 0.002 & 0.363 \\
\hline Oncoarray & 380.157 & $<0.001^{\star}$ & 377.314 & $<0.001^{\star}$ & 0.005 & 0.141 \\
\hline iCOGS & 402.683 & $<0.001^{\star}$ & 402.556 & $<0.001^{\star}$ & 0.001 & 0.763 \\
\hline GWAS & 316.552 & 0.136 & 315.444 & 0.137 & -0.009 & 0.314 \\
\hline \multicolumn{7}{|l|}{ ER- } \\
\hline Combination & 417.539 & $<0.001^{\star}$ & 414.293 & $<0.001^{\star}$ & 0.006 & 0.134 \\
\hline Oncoarray & 339.408 & $0.024^{\star}$ & 337.396 & $0.026^{\star}$ & 0.006 & 0.190 \\
\hline iCOGS & 323.401 & 0.086 & 323.248 & 0.081 & 0.002 & 0.712 \\
\hline GWAS & 326.926 & 0.067 & 325.029 & 0.071 & 0.010 & 0.195 \\
\hline \multicolumn{7}{|c|}{$\begin{array}{l}\text { Breast cancer, overall breast cancer risk; ER+, estrogen receptor (ER)-positive breast cancer risk; ER-, } \\
\text { ER-negative breast cancer risk; Four databases: (1) the OncoArray Consortium; (2) Collaborative } \\
\text { Oncological Gene-environment Study (iCOGS); (3) } 11 \text { other breast cancer genome-wide association } \\
\text { studies (GWAS); (4) Combination of above three databases. }\end{array}$} \\
\hline
\end{tabular}

In the combined dataset, genetically predicted higher educational attainment tended to decrease the risk of ER-negative breast cancer (ER-) (Fig. 1). Using random-effects IVW method, each additional SD higher education was associated with a $27 \%$ lower risk of ER- (OR 0.73, 95\% $\mathrm{Cl}[0.64,0.84]$; P-value $<0.001)$. Supplementary Fig. 7 shows the forest plot of 291 SNPs associated with education and their risk of ER-. As expected, the associations were consistent with sensitivity analyses using the weighted mode (OR 0.76 , $95 \% \mathrm{Cl}[0.64,0.91] ;$ P-value $=0.002)$ and MR-Egger method (OR 0.49, 95\% Cl [0.29, 0.84]; P-value = 0.01), but provided less precise estimates than the IVW method. The scatter plots are shown in Supplementary Fig. 8. In the heterogeneity test, we found that no single SNP was significantly contributing to the overall effect of education on ER- (Supplementary Fig. 9). The results of the MR-Egger test suggested that there is 
no directional pleiotropy (Table 2), and the results for MR-Egger are consistent with the IVW analysis.

Similar results were obtained using the Oncoarray, iCOGS, and GWAS datasets (Fig. 1 and Supplementary Fig. 11-22). In addition, we also found a very weak causal association for overall breast cancer (OR 0.91, $95 \% \mathrm{Cl}[0.83,0.997]$, P-value $=0.042)$ in combined database but a null causal association in other three datasets (Fig. 1). However, we observed a null causal association for ER+ (OR 0.94, 95\% Cl [0.85, 1.04]; Pvalue $=0.26)($ Fig. 1) in all databases. The results for the pleiotropy test are listed in Table 2.

\section{Causal effect of education on potential risk factors of breast cancer}

To identify the underlying mechanism of the association between level of education and ER-, we investigated whether several potential cancer risk factors play a role. Table 3 shows that each SD higher level of education was associated with $32 \%$ lower odds of smoking, 1.89 times higher odds of smoking cessation among smokers, less smoking intensity $(-2.26[-3.48,-0.65]$ cigarettes per day), 0.35 lower body mass index, 0.13 lower waist-to-hip ratio, 0.35 higher height, 0.09 higher hip circumference, 0.15 $\mathrm{mmol} / \mathrm{L}$ lower triglycerides, and $0.16 \mathrm{mmol} / \mathrm{L}$ higher $\mathrm{HDL}$-cholesterol $(P<0.05)$. 
Table 3

Causal effects of educational attainment on risk factors of breast cancer (potential mediators)

Outcome nsnp $\begin{aligned} & \text { Causal } \\ & \text { effect }\end{aligned} \quad$ Pl Pval Q_pval $\begin{gathered}\text { Egger's } \\ \text { test } \\ \text { pval }\end{gathered}$

\section{Lipids}

HDL cholesterol

227

0.16

$[0.10,0.22]$

$\begin{array}{ll}<.01 * & < \\ 0.01 *\end{array}$

0.76

LDL cholesterol

$227-0.08$

$[-0.15,-0.01]$

$0.02^{*}<$

$0.01 *$

0.62

Total cholesterol

227

$227-0.05 \quad[-0.12,0.02] \quad 0.16 \quad \begin{array}{lll}< & 0.78\end{array}$

Triglycerides

227

$-0.15$

$[-0.21,-0.08]$

$\dot{0.01 *} \quad \dot{0.01 *}$

0.77

\section{Anthropometric measures}

Hip circumference

$247 \quad 0.09$

$[0.01,0.16]$

$0.02^{*}$

$<$

$0.01 *$

0.35

Waist circumference

$247 \quad-0.06$

$[-0.13,0.01]$

0.07

$0.01 *$

0.57

Waist-to-hip ratio

247

$-0.13$

$[-0.19,-0.06]$

$0.01^{*}$

$0.01^{*}$

0.90

Height

247

0.10

$[0.02,0.18]$

$0.02^{\star}$

$0.01 *$

0.50

Body mass index (BMI)

307

$07-0.35$

$[-0.40,-0.29]$

$<$

0.01 * 0.01 *

0.74

Sleep duration

306

$0.04 \quad[0,0.09]$

0.07

<.01*

0.48

\section{Smoke}

Cigarettes smoked per day

$247 \quad-2.26$

$[-3.13,-1.38]$

<.01*

0.41

0.95

Ever vs never smoked

247

0.68

$[0.59,0.79]$

<.01*

0.01 *

0.87

Former vs current smoker

$247 \quad 1.89$

$[1.59,2.24]$

<.01*

0.21

0.55

\section{Physical activities}

Types of physical activity in last 4 weeks: Light DIY (eg: pruning, watering the lawn)
$307 \quad 1.13$

[1.13,1.16]
0.09 


\begin{tabular}{|c|c|c|c|c|c|c|}
\hline Outcome & nsnp & $\begin{array}{l}\text { Causal } \\
\text { effect }\end{array}$ & $\mathrm{Cl}$ & Pval & Q_pval & $\begin{array}{l}\text { Egger's } \\
\text { test } \\
\text { pval }\end{array}$ \\
\hline $\begin{array}{l}\text { Types of physical activity in last } 4 \\
\text { weeks: Heavy DIY (eg: weeding, lawn } \\
\text { mowing, carpentry, digging) }\end{array}$ & 307 & 1.08 & {$[1.07,1.11]$} & $<0.01 *$ & $<.01^{*}$ & 0.30 \\
\hline $\begin{array}{l}\text { Types of physical activity in last } 4 \\
\text { weeks: Strenuous sports }\end{array}$ & 307 & 1.06 & {$[1.05,1.07]$} & $<.01 *$ & $<.01 *$ & 0.53 \\
\hline $\begin{array}{l}\text { Types of physical activity in last } 4 \\
\text { weeks: Walking for pleasure (not as a } \\
\text { means of transport) }\end{array}$ & 307 & 1.12 & {$[1.11,1.13]$} & $<.01^{*}$ & $\dot{0} .01^{*}$ & 0.55 \\
\hline $\begin{array}{l}\text { Types of physical activity in last } 4 \\
\text { weeks: Other exercises (eg: swimming, } \\
\text { cycling, keep fit, bowling) }\end{array}$ & 307 & 1.16 & {$[1.15,1.17]$} & $\dot{0.01 *}$ & $\dot{0.01 *}$ & 0.97 \\
\hline $\begin{array}{l}\text { Types of physical activity in last } 4 \\
\text { weeks: None of the above }\end{array}$ & 307 & 0.94 & {$[0.94,0.95]$} & $<.01 *$ & $<.01 *$ & 0.06 \\
\hline Time spent doing light physical activity & 307 & -1.7 & {$[-2.31,1.51]$} & $<.01 *$ & 0.47 & 0.18 \\
\hline $\begin{array}{l}\text { Time spent doing moderate physical } \\
\text { activity }\end{array}$ & 307 & -0.06 & {$[-0.14,0.02]$} & 0.17 & 0.09 & 0.17 \\
\hline $\begin{array}{l}\text { Time spent doing vigorous physical } \\
\text { activity }\end{array}$ & 307 & -0.23 & {$[-0.31,0.51]$} & $<.01 *$ & $0.01 *$ & 0.40 \\
\hline $\begin{array}{l}\text { Number of days/week of moderate } \\
\text { physical activity } 10+\text { minutes }\end{array}$ & 307 & -0.24 & {$[-0.31,-0.16]$} & $<.01 *$ & $<.01^{*}$ & 0.27 \\
\hline $\begin{array}{l}\text { Number of days/week of vigorous } \\
\text { physical activity } 10+\text { minutes }\end{array}$ & 307 & -0.07 & {$[-0.13,-0.01]$} & $0.03^{*}$ & $<.01 *$ & 0.15 \\
\hline
\end{tabular}

In addition, physical activity performed during the last four weeks has a causal effect on the risk of EREach SD higher level of education increased $13 \%$ odds of light (e.g. pruning, watering the lawn) and $8 \%$ heavy DIY activities (e.g. weeding, lawn mowing, carpentry, digging). It was also associated with $6 \%$ higher odds of strenuous sports, $12 \%$ leisure walking, and $16 \%$ other activities (e.g. swimming, cycling, keeping fit, bowling) in the last four weeks. In addition, ER- also decreased with over $10 \mathrm{~min}$ of moderate $(0.24$ days/week) and vigorous (0.07 days/week) physical activity.

The results of other sensitivity analysis methods for the above causal associations are shown in Supplementary Figs. 1-2. All the samples used provided sufficient statistical power (100\%) to identify causal effects.

\section{Causal effect of potential mediators on the risk of breast cancer}


MR was performed to evaluate the causal effects of the previously described potential mediators on the risk of breast cancer. Table 4 shows the causal effects of potential mediators on ER- in different datasets. Higher HDL cholesterol is causally associated higher risk of ER- $(\mathrm{OR} 1.10,95 \% \mathrm{Cl}[1.04,1.15], \mathrm{P}$-value $=$ 0.001 ). Higher triglycerides, $\mathrm{BMI}$, and waist circumference were associated with $8 \%, 11 \%$, and $52 \%$ lower risk of ER-, respectively. Light and heavy DIY activities, leisure walking, and other exercises (e.g. swimming, cycling, keeping fit bowling) lowered the risk of ER- by $34 \%, 49 \%$, and $46 \%$, respectively. Increasing the number of days/week of moderate activity for at least 10 min can also decrease the risk of ER-. In conclusion, HDL cholesterol, triglycerides, BMI, waist circumference, smoking, and types and times of moderate activity above 10 minutes are mediators in the causal pathway from educational attainment to ER- (Fig. 2). Other potential mediators were causally associated with the risk of overall breast cancer or ER+, but not ER- (Supplementary Figs. 3-6). 
Table 4

Causal effects of potential mediators on ER-negative breast cancer risk (ER-)

\begin{tabular}{|c|c|c|c|c|c|c|}
\hline Exposure & nsnp & OR & $95 \% \mathrm{Cl}$ & pval & Q_pval & $\begin{array}{l}\text { Egger's test } \\
\text { pval }\end{array}$ \\
\hline \multicolumn{7}{|c|}{ HDL cholesterol } \\
\hline Combination & 296 & 1.10 & {$[1.04,1.15]$} & $0.00 *$ & 0.00 & 0.84 \\
\hline Oncoarray & 296 & 1.11 & {$[1.04,1.18]$} & $0.00 *$ & 0.00 & 0.40 \\
\hline iCOGS & 296 & 1.11 & {$[1.03,1.19]$} & $0.01 *$ & 0.02 & 0.76 \\
\hline GWAS & 296 & 1.05 & {$[0.96,1.16]$} & 0.29 & 0.50 & 0.63 \\
\hline \multicolumn{7}{|l|}{ Triglycerides } \\
\hline Combination & 249 & 0.95 & {$[0.89,1.01]$} & 0.09 & 0.00 & 0.49 \\
\hline Oncoarray & 249 & 0.92 & {$[0.85,0.99]$} & $0.03^{*}$ & 0.00 & 0.96 \\
\hline iCOGS & 249 & 0.99 & {$[0.9,1.08]$} & 0.83 & 0.00 & 0.73 \\
\hline GWAS & 249 & 0.94 & {$[0.75,1.18]$} & 0.61 & 0.00 & 0.09 \\
\hline \multicolumn{7}{|c|}{ Body mass index (BMI) } \\
\hline Combination & 619 & 0.89 & {$[0.82,0.96]$} & $0.00 *$ & 0.00 & 0.00 \\
\hline Oncoarray & 620 & 0.87 & {$[0.79,0.96]$} & $0.01^{\star}$ & 0.01 & 0.00 \\
\hline iCOGS & 617 & 0.56 & {$[0.41,0.77]$} & $0.00 *$ & 0.00 & 0.00 \\
\hline GWAS & 620 & 0.54 & {$[0.35,0.84]$} & $0.01^{*}$ & 0.01 & 0.01 \\
\hline \multicolumn{7}{|c|}{ Waist circumference } \\
\hline Combination & 60 & 0.98 & {$[0.83,1.14]$} & 0.77 & 0.00 & 0.20 \\
\hline Oncoarray & 60 & 0.48 & {$[0.24,0.96]$} & $0.04 *$ & 0.01 & 0.04 \\
\hline iCOGS & 60 & 0.86 & {$[0.7,1.07]$} & 0.18 & 0.09 & 0.80 \\
\hline GWAS & 60 & 1.30 & {$[0.99,1.7]$} & 0.06 & 0.03 & 0.94 \\
\hline \multicolumn{7}{|c|}{ Waist-to-hip ratio } \\
\hline Combination & 51 & 0.93 & {$[0.8,1.08]$} & 0.34 & 0.00 & 0.40 \\
\hline Oncoarray & 51 & 0.95 & {$[0.78,1.15]$} & 0.57 & 0.00 & 0.15 \\
\hline iCOGS & 51 & 0.86 & {$[0.71,1.05]$} & 0.13 & 0.21 & 0.67 \\
\hline
\end{tabular}

Four databases: (1) the OncoArray Consortium; (2) Collaborative Oncological Gene-environment Study (iCOGS); (3) 11 other breast cancer genome-wide association studies (GWAS); (4) Combination of above three databases. 


\begin{tabular}{|c|c|c|c|c|c|c|}
\hline Exposure & nsnp & OR & $95 \% \mathrm{Cl}$ & pval & Q_pval & $\begin{array}{l}\text { Egger's test } \\
\text { pval }\end{array}$ \\
\hline GWAS & 51 & 1.02 & {$[0.76,1.36]$} & 0.89 & 0.05 & 0.79 \\
\hline \multicolumn{7}{|c|}{ Cigarettes smoked per day } \\
\hline Combination & 6 & 1.00 & {$[0.99,1.01]$} & 0.75 & 0.79 & 0.84 \\
\hline Oncoarray & 6 & 1.00 & {$[0.98,1.01]$} & 0.82 & 0.87 & 0.75 \\
\hline iCOGS & 6 & 1.00 & {$[0.99,1.02]$} & 0.45 & 0.95 & 0.77 \\
\hline GWAS & 6 & 0.98 & {$[0.95,1.02]$} & 0.41 & 0.01 & 0.85 \\
\hline \multicolumn{7}{|c|}{ Ever vs never smoked } \\
\hline Combination & 5 & 0.87 & {$[0.76,0.99]$} & $0.04^{\star}$ & 0.67 & 0.57 \\
\hline Oncoarray & 5 & 0.86 & {$[0.68,1.1]$} & 0.22 & 0.45 & 0.56 \\
\hline iCOGS & 5 & 1.01 & {$[0.92,1.1]$} & 0.86 & 0.99 & 0.88 \\
\hline GWAS & 5 & 0.65 & {$[0.42,1.01]$} & 0.05 & 0.58 & 0.54 \\
\hline \multicolumn{7}{|c|}{ Former vs current smoker } \\
\hline Combination & 7 & 1.06 & {$[0.97,1.16]$} & 0.17 & 0.55 & 0.26 \\
\hline Oncoarray & 7 & 1.03 & {$[0.89,1.19]$} & 0.69 & 0.29 & 0.09 \\
\hline iCOGS & 7 & 1.09 & {$[0.99,1.21]$} & 0.09 & 0.89 & 0.58 \\
\hline GWAS & 7 & 1.09 & {$[0.87,1.36]$} & 0.45 & 0.22 & 0.83 \\
\hline \multicolumn{7}{|c|}{ Types: Light DIY } \\
\hline Combination & 76 & 0.66 & {$[0.33,1.31]$} & 0.23 & 0.00 & 0.84 \\
\hline Oncoarray & 76 & 1.11 & {$[0.55,2.24]$} & 0.77 & 0.23 & 0.76 \\
\hline iCOGS & 76 & 0.73 & {$[0.26,2.02]$} & 0.54 & 0.02 & 0.66 \\
\hline GWAS & 75 & 0.11 & {$[0.03,0.35]$} & $0.00 *$ & 0.49 & 0.40 \\
\hline \multicolumn{7}{|c|}{ Types: Heavy DIY } \\
\hline Combination & 111 & 2.5 & {$[1.82,5]$} & $0.01^{*}$ & 0.00 & 0.01 \\
\hline Oncoarray & 111 & 2.5 & {$[2.26,5]$} & $0.01^{\star}$ & 0.00 & 0.01 \\
\hline iCOGS & 111 & 1.90 & {$[0.78,4.63]$} & 0.16 & 0.01 & 0.52 \\
\hline GWAS & 110 & 0.35 & {$[0.13,0.95]$} & $0.04^{*}$ & 0.01 & 0.12 \\
\hline
\end{tabular}




\begin{tabular}{|c|c|c|c|c|c|c|}
\hline Exposure & nsnp & OR & $95 \% \mathrm{Cl}$ & pval & Q_pval & $\begin{array}{l}\text { Egger's test } \\
\text { pval }\end{array}$ \\
\hline \multicolumn{7}{|c|}{ Types: Walking for pleasure } \\
\hline Combination & 67 & 0.51 & {$[0.29,0.91]$} & $0.02^{\star}$ & 0.42 & 0.56 \\
\hline Oncoarray & 67 & 0.38 & {$[0.17,0.86]$} & $0.02 *$ & 0.98 & 0.43 \\
\hline iCOGS & 67 & 0.53 & {$[0.18,1.61]$} & 0.27 & 0.17 & 0.79 \\
\hline GWAS & 67 & 1.12 & {$[0.28,4.49]$} & 0.87 & 0.33 & 0.78 \\
\hline \multicolumn{7}{|c|}{ Types: Strenuous sports } \\
\hline Combination & 41 & 0.35 & {$[0.08,1.5]$} & 0.16 & 0.01 & 0.28 \\
\hline Oncoarray & 41 & 0.66 & {$[0.1,4.49]$} & 0.67 & 0.05 & 0.78 \\
\hline iCOGS & 41 & 0.19 & {$[0.02,1.43]$} & 0.11 & 0.38 & 0.27 \\
\hline GWAS & 41 & 0.19 & {$[0.01,3.14]$} & 0.24 & 0.70 & 0.23 \\
\hline \multicolumn{7}{|c|}{ Type: Other exercises } \\
\hline Combination & 92 & 0.54 & {$[0.34,0.85]$} & $0.01^{*}$ & 0.09 & 0.98 \\
\hline Oncoarray & 92 & 0.53 & {$[0.28,1.01]$} & 0.05 & 0.16 & 0.53 \\
\hline iCOGS & 92 & 0.60 & {$[0.27,1.34]$} & 0.21 & 0.46 & 0.75 \\
\hline GWAS & 92 & 0.45 & {$[0.15,1.33]$} & 0.15 & 0.48 & 0.44 \\
\hline \multicolumn{7}{|c|}{ Types: None of the above } \\
\hline Combination & 46 & 3.94 & {$[0.72,21.48]$} & 0.11 & 0.02 & 0.56 \\
\hline Oncoarray & 46 & 1.76 & {$[0.24,12.78]$} & 0.58 & 0.07 & 0.49 \\
\hline iCOGS & 46 & 4.27 & {$[0.25,72.47]$} & 0.32 & 0.07 & 0.36 \\
\hline GWAS & 46 & 37.78 & {$[1.55,923.56]$} & $0.03^{\star}$ & 0.67 & 0.09 \\
\hline \multicolumn{7}{|c|}{ Time spent doing light physical activity } \\
\hline Combination & 24 & 1.00 & {$[1,1]$} & 0.12 & 0.33 & 0.63 \\
\hline Oncoarray & 24 & 1.00 & {$[1,1]$} & 0.23 & 0.62 & 0.96 \\
\hline iCOGS & 24 & 1.00 & {$[1,1.01]$} & 0.19 & 0.55 & 0.17 \\
\hline GWAS & 23 & 1.00 & {$[0.99,1.01]$} & 0.81 & 0.37 & 0.46 \\
\hline
\end{tabular}

Four databases: (1) the OncoArray Consortium; (2) Collaborative Oncological Gene-environment Study (iCOGS); (3) 11 other breast cancer genome-wide association studies (GWAS); (4) Combination of above three databases. 


\begin{tabular}{|c|c|c|c|c|c|c|}
\hline Exposure & nsnp & OR & $95 \% \mathrm{Cl}$ & pval & Q_pval & $\begin{array}{l}\text { Egger's test } \\
\text { pval }\end{array}$ \\
\hline \multicolumn{7}{|c|}{$\begin{array}{l}\text { Time spent doing vigorous physical } \\
\text { activity }\end{array}$} \\
\hline Combination & 7 & 1.00 & {$[0.64,1.56]$} & 1.00 & 0.00 & 0.20 \\
\hline Oncoarray & 7 & 1.06 & {$[0.69,1.63]$} & 0.79 & 0.12 & 0.07 \\
\hline iCOGS & 7 & 0.94 & {$[0.48,1.85]$} & 0.87 & 0.02 & 0.28 \\
\hline GWAS & 7 & 0.93 & {$[0.35,2.47]$} & 0.88 & 0.02 & 0.64 \\
\hline \multicolumn{7}{|c|}{ Number of days/week of moderate activity $10+$ minutes } \\
\hline Combination & 74 & 0.95 & {$[0.85,1.06]$} & 0.35 & 0.19 & 0.18 \\
\hline Oncoarray & 74 & 1.08 & {$[0.94,1.24]$} & 0.26 & 0.74 & 0.49 \\
\hline iCOGS & 74 & 0.88 & {$[0.74,1.04]$} & 0.13 & 0.68 & 0.06 \\
\hline GWAS & 74 & 0.76 & {$[0.59,0.97]$} & $0.03 *$ & 0.51 & 0.68 \\
\hline \multicolumn{7}{|c|}{$\begin{array}{l}\text { Four databases: (1) the OncoArray Consortium; (2) Collaborative Oncological Gene-environment Study } \\
\text { (iCOGS); (3) } 11 \text { other breast cancer genome-wide association studies (GWAS); (4) Combination of } \\
\text { above three databases. }\end{array}$} \\
\hline
\end{tabular}

\section{Discussion}

Our study showed that each SD higher education level decreased the risk of ER- by $22 \%$ (OR $0.77,95 \% \mathrm{Cl}$ $[0.6,0.984] ; P=0.004)$. However, no causal association was found between overall breast cancer and ER + risk, consistent with the results of sensitivity analyses. HDL cholesterol, triglycerides, BMI, waist circumference, and types and times of moderate activity above $10 \mathrm{~min}$ were identified as mediators in the causal pathway from educational attainment to ER-

A large meta-analysis including more than 10 million women found that a high degree of education may be associated with a higher risk of breast cancer. In addition, menopausal age, alcohol consumption, and hormone therapy may mediate this causal effect to a certain extent [25]. However, this may be hampered by underlying sources of bias (e.g., unmeasured confounding and reverse causation). A cohort study of 3092 individuals born in Limache Hospital between 1974 and 1978 showed that poor education may be associated with a poor lipid profile in women [41]. Consistently with this study, a two-sample MR study involving over 400,000 participants [26] showed that increased LDL-cholesterol levels were associated with a higher risk of breast cancer (OR 1.09, 95\% $\mathrm{Cl}[1.02,1.18]$, P-value $=0.020)$ and $E R+$ breast cancer (OR 1.14 [1.05-1.24], P-value = 0.004). Individuals with genetically higher HDL-cholesterol levels were at increased risk of developing ER+ (OR 1.13 [1.01-1.26], P-value = 0.037). Higher HDL-cholesterol and lower triglyceride levels were found to be determinants of ER-. However, HDL- and LDL-cholesterol and triglyceride levels had no nominally significant association with either overall breast cancer risk or ERbreast cancer. A limitation of their study is the lack of stratification of women by menopausal status. 
Endocrine changes during menopause may alter the lipid composition and the interaction with breast tissue. For example, a meta-analysis of observational studies found a negative association between HDL cholesterol and breast cancer only exists in postmenopausal women, but not in premenopausal women. [27] A further limitation of this study is that the effect of age at menopause and hormone therapy were not considered. Further studies are warranted to investigate the role of menopausal status in the causal pathway from education level to breast cancer.

A cross-sectional study indicated that low levels of education were independently associated with obesity [42]. Consistent with our results, another MR study suggested that increased BMI could lower breast cancer survival for ER + breast cancer patients. [28] However, their results indicated that BMI had no causal effect on ER- breast cancer. A limitation of their analysis is that there might be a selection bias from the genetic variants associated with these confounders for the subpopulation of breast cancer patients. This is due to conditioning on a collider and is referred to as selection bias, which indicates the need to select a representative population for MR analysis. A simulation study found that only when the selection effect is large, the selection bias will significantly affect the estimation of the causal effect and the type 1 error rate. [29].

In accordance with our results, another MR study indicated that accelerometer-measured physical activity was negatively associated with the risk of breast cancer. Multiple biological mechanisms have been proposed to explain the potential beneficial role of physical activity on breast cancer development. Physical activity can reduce circulating insulin and insulin-like growth factor levels, promote cell proliferation in breast tissue and prevent cancer development in these areas. High levels of physical activity also reduced circulating levels of estradiol and increased levels of sex hormone-binding globulin [37], which are risk factors for breast cancer. However, few studies investigate the causal relationship between level of education and physical activity.

Our study has several important advantages. We conducted an MR study to investigate the causal relationship between the level of education and breast cancer. Participants were grouped according to their randomly assigned genotypes, similarly to randomized control trials. The MR design avoids the interference of reverse causation and potential confounding factors that are common in conventional observational studies. The large sample size of summary datasets improves the statistical power and estimate the causal effect with high precision. The strong instrumental variables ( $F$ statistics $>10)$ [45] compensated for the weak instrumental bias.

In addition, our study also has several limitations. First, all the participants included in our study were of European descent. Thus, it is unknown whether our findings can be extended to other ethnicities. In addition, the InSIDE assumption in the MR-Egger test remains a limitation. In the InSIDE assumption, the effect of genetic variants on the exposure is independent of the direct effects of the genetic variants on the outcome, which is difficult to evaluate. Further studies are warranted to investigate the role of menopausal status in the causal pathway between the level of education and breast cancer. 


\section{Conclusion}

In conclusion, our present Mendelian randomization study provided strong evidence to suggest that higher educational attainment played a causal role in lowering the risk of breast cancer. A low level of education is a causal risk factor in the development of ER- as it is associated with a poor lipid profile, anthropometric measurements, smoking, and types of physical activity.

\section{Declarations}

\section{Novelty \& Impact Statements}

Using several publicly available summary-level genetic data from large-sized consortia, we conducted a Mendelian randomization (MR) study and found that genetic susceptibility towards the level of education was causally associated with the risk of estrogen receptor (ER)- partially by altering waist circumference, body mass index (BMI), levels of triglycerides and $\mathrm{HDL}$, smoking, and physical activity.

\section{Funding}

This work was supported by the National Natural Science Foundation of China (grant number 81773547 and 82003557), Shandong Provincial Natural Science Foundation of China (ZR2019ZD02) and Shandong Province Major Science and Technology Innovation Project (2018CXGC1210).

\section{Conflict of Interest}

None declared.

\section{Declarations of interest: None}

\section{Availability of data and material}

SSGAC, Social Science Genetic Association Consortium; GLGC, Global Lipids Genetics Consortium; BCAC, Breast Cancer Association Consortium; MAGIC, Meta-Analyses of Glucose and Insulin-related traits Consortium; MRC-IEU, MRC Integrative Epidemiology Unit; TAG, Tobacco and Genetics consortium.

\section{Code availability}

The R package TwoSampleMR (v0.5.1) was used for all MR methods (version 3.6.3). Power calculations on MR analysis were performed in http://cnsgenomics.com/shiny/mRnd/.

\section{Authors' contributions:}

Fuzhong Xue, Hongkai Li and Lei Hou: conceptualization, methodology and software; Yuanyuan Yu and Xiaoru Sun: data curation and writing original draft; Xinhui Liu, Ran Yan and Yifan Yu: visualization and research; Sijia Wu and Yina He: supervision; Yutong Wu and Li He: software and validation; Hongkai Li and Lei Hou: writing, reviewing and editing. 


\section{References}

1. Harbeck N, Gnant M. Breast cancer Lancet. 2017;389:1134-50.

2. Global Burden of Disease Cancer Collaboration. Global, regional, and national cancer incidence, mortality, years of life lost, years lived with disability, and disability-adjusted life-years for 32 cancer groups, 1990 to 2015: a systematic analysis for the global burden of disease study. JAMA Oncol. 2017;3:524-48.

3. DeSantis C, Ma J, Bryan L, Jemal A. Breast cancer statistics, 2013. CA Cancer J Clin. 2014;64(1):5262.

4. DeSantis C, Howlader N, Cronin KA, Jemal A. Breast cancer incidence rates in U.S. women are no longer declining. Cancer Epidemiol Bio-markers Prev. 2011;20:733-49.

5. Menvielle $\mathrm{G}$, Kunst $\mathrm{AE}$, van Gils $\mathrm{CH}$, et al. The contribution of risk factors to the higher incidence of invasive and in situ breast cancers in women with higher levels of education in the European prospective investigation into cancer and nutrition. Am J Epidemiol. 2011;173:26-37.

6. Goldberg M, Calderon-Margalit R, Paltiel $\mathrm{O}$, et al. Socioeconomic disparities in breast cancer incidence and survival among parous women: findings from a population-based cohort, 1964-2008. BMC Cancer. 2015;15:921.

7. Carlsen K, Hoybye MT, Dalton SO, Tjonneland A. Social inequality and incidence of and survival from breast cancer in a population-based study in Denmark, 1994-2003. Eur J Cancer. 2008;44:19962002.

8. Hajian-Tilaki K, Kaveh-Ahangar T, Hajian-Tilaki E. Is educational level associated with breast cancer risk in Iranian women? Breast cancer (Tokyo Japan). 2012;19(1):64-70. https://doi.org/10.1007/s12282-011-0273-6.

9. Bjerkaas E, Parajuli R, Engeland A, Maskarinec G, Weiderpass E, Gram IT. Social inequalities and smoking-associated breast cancer-results from a prospective cohort study. Prev Med. 2015;73:1259.

10. Hussain SK, Altieri A, Sundquist J, Hemminki K. Influence of education level on breast cancer risk and survival in Sweden between 1990 and 2004. Int J Cancer. 2008;122:165-9.

11. Hajian-Tilaki K, Kaveh-Ahangar T, Hajian-Tilaki E. Is educational level associated with breast cancer risk in Iranian women? Breast Cancer. 2012;19(1):64-70. doi:10.1007/s12282-011-0273-6.

12. Burgess S, Small DS, Thompson SG. A review of instrumental variable estimators for Mendelian randomization. Stat Methods Med Res. 2017;26(5):2333-55. doi:10.1177/0962280215597579.

13. Burgess S, Butterworth A, Thompson SG.. Mendelian Randomization Analysis With Multiple Genetic Variants Using Summarized Data [J]. Genet Epidemiol, 2013, 37(7):658-65.

14. Zhou H, Zhang Y, Liu J, et al. Education and lung cancer: a Mendelian randomization study. Int J Epidemiol. 2019;48(3):743-50. doi:10.1093/ije/dyz121.

15. Gill D, Efstathiadou A, Cawood K, Tzoulaki I, Dehghan A. Education protects against coronary heart disease and stroke independently of cognitive function: evidence from Mendelian randomization. Int 
J Epidemiol. 2019;48(5):1468-77. doi:10.1093/ije/dyz200.

16. Mountjoy E, Davies NM, Plotnikov D, et al. Education and myopia: assessing the direction of causality by mendelian randomisation [published correction appears in BMJ. 2018 Jul 4;362:k2932]. BMJ. 2018;361:k2022. Published 2018 Jun 6. doi:10.1136/bmj.k2022.

17. Okbay A, Beauchamp JP, Fontana MA, et al. Genome-wide association study identifies 74 loci associated with educational attainment. Nature. 2016;533:539.

18. Shungin D, Winkler TW, Croteau-Chonka DC, et al. New genetic loci link adipose and insulin biology to body fat distribution. Nature. 2015;518(7538):187-96. doi:10.1038/nature14132.

19. Andersson U, Lewander T, Gunne LM. Rhythmic jaw movements induced by ethanol in rats. Pharmacol Toxicol. 1989;64(4):378-9. doi:10.1111/j.1600-0773.1989.tb00669.x.

20. Twarog FJ, Picone FJ, Strunk RS, So J, Colten HR. Immediate hypersensitivity to cockroach. Isolation and purification of the major antigens. J Allergy Clin Immunol. 1977;59(2):154-60. doi:10.1016/0091-6749(77)90218-4.

21. Tobacco and Genetics Consortium. Genome-wide meta-analyses identify multiple loci associated with smoking behavior. Nat Genet. 2010;42(5):441-7. doi:10.1038/ng.571.

22. Amos $\mathrm{Cl}$, et al. The OncoArray Consortium: a network for understanding the genetic architecture of common cancers. Cancer Epidemiol Biomarkers Prev. 2017;26:126-35.

23. Michailidou $\mathrm{K}$, et al. Large-scale genotyping identifes 41 new loci associated with breast cancer risk. Nat Genet. 2013;45:353-61.

24. Burgess S, Davies NM, Thompson SG. Bias due to participant overlap in two-sample Mendelian randomization. Genet Epidemiol. 2016;40(7):597-608. doi:10.1002/gepi.21998.

25. Dong APA, Jia-Yi PhD1; Qin. Li-Qiang PhD2 Education level and breast cancer incidence, Menopause: January 2020 - Volume 27 - Issue 1 - p 113-118 doi: 10.1097/GME.0000000000001425.

26. Nowak C, Ärnlöv J. A Mendelian randomization study of the effects of blood lipids on breast cancer risk. Nat Commun. 2018;9(1):3957. doi:10.1038/s41467-018-06467-9. Published 2018 Sep 27.

27. $\mathrm{Ni} \mathrm{H}$, Liu H, Gao R. Serum lipids and breast cancer risk: a meta-analysis of prospective cohort studies. PloS ONE. 2015;10:e0142669.

28. Guo Q, Burgess S, Turman C, et al. Body mass index and breast cancer survival: a Mendelian randomization analysis. Int J Epidemiol. 2017;46(6):1814-22. doi:10.1093/ije/dyx131.

29. Gkatzionis A, Burgess S. Contextualizing selection bias in Mendelian randomization: how bad is it likely to be? Int J Epidemiol. 2019;48(3):691-701. doi:10.1093/ije/dyy202.

30. Papadimitriou N, Dimou N, Tsilidis KK, et al. Physical activity and risks of breast and colorectal cancer: a Mendelian randomisation analysis. Nat Commun. 2020;11(1):597. Published 2020 Jan 30. doi:10.1038/s41467-020-14389-8.

31. Bowers LW, Rossi EL, O'Flanagan CH, deGraffenried LA, Hursting SD. The role of the insulin/igf system in cancer: lessons learned from clinical trials and the energy balance-cancer link. Frontiers in Endocrinology 6, https://doi.org/10.3389/fendo.2015.00077 (2015). Return to ref 23 in article. 
32. Pollak M. Insulin and insulin-like growth factor signalling in neoplasia. Nat Rev Cancer. 2008;8:915.

33. Shu X, et al. Associations of obesity and circulating insulin and glucose with breast cancer risk: a Mendelian randomization analysis. Int J Epidemiol. 2018;48:795-806.

34. Murphy $\mathrm{N}$, et al. A nested case-control study of metabolically defined body size phenotypes and risk of colorectal cancer in the European Prospective Investigation into Cancer and Nutrition (EPIC). PLoS Med. 2016;13:e1001988.

35. The Endogenous H, Breast C, Collaborative G. Insulin-like growth factor 1 (IGF1), IGF binding protein 3 (IGFBP3), and breast cancer risk: pooled individual data analysis of 17 prospective studies. Lancet Oncol. 2010;11:530-42.

36. McTiernan A, et al. Effect of exercise on serum estrogens in postmenopausal women: a 12-month randomized clinical trial. Cancer Res. 2004;64:2923-8.

37. Liedtke S, et al. Physical activity and endogenous sex hormones in postmenopausal women: to what extent are observed associations confounded or modified by BMI? Cancer Causes Control. $2011 ; 22: 81-9$.

38. Bertone-Johnson ER, Tworoger SS, Hankinson SE. Recreational physical activity and steroid hormone levels in postmenopausal women. Am J Epidemiol. 2009;170:1095-104.

39. Endogenous Hormones and Breast Cancer Collaborative Group. Sex hormones and risk of breast cancer in premenopausal women: a collaborative reanalysis of individual participant data from seven prospective studies. Lancet Oncol. 2013;14:1009-19.

40. The Endogenous Hormones Breast Cancer Collaborative Group. Endogenous sex hormones and breast cancer in postmenopausal women: reanalysis of nine prospective studies. JNCl: J Natl Cancer Inst. 2002;94:606-16.

41. Lara M, Amigo H. Association between education and blood lipid levels as income increases over a decade: a cohort study. BMC Public Health. 2018;18(1):286. doi:10.1186/s12889-018-5185-3. Published 2018 Feb 27.

42. Sánchez CN, Maddalena N, Penalba M, Quarleri M, Torres V, Wachs A. Relación entre nivel de instrucción y exceso de peso en pacientes de consulta externa. Estudio transversal [Relationship between level of education and overweight in outpatients. A transversal study]. Medicina. 2017;77(4):291-6.

43. Lee, J. J., Wedow, R., Okbay, A., Kong, E., Maghzian, O., Zacher, M., Nguyen-Viet,T. A., Bowers, P., Sidorenko, J., Karlsson Linnér, R., Fontana, M. A., Kundu, T.,Lee, C., Li, H., Li, R., Royer, R., Timshel, P. N., Walters, R. K., Willoughby, E.A., Yengo, L., ... Cesarini, D. (2018). Gene discovery and polygenic prediction from a genome-wide association study of educational attainment in 1.1 million individuals.Nature genetics, 50(8), 1112-1121. https://doi.org/10.1038/s41588-018-0147-3.

44. Hou L, Li H, Si S, Yu Y, Sun X, Liu X, Yan R, Yu Y, Wang C, Yang F, Wang Q, Xue F. Exploring the causal pathway from bilirubin to CVD and diabetes in the UK biobank cohort study: Observational findings and Mendelian randomization studies. Atherosclerosis. 2021;320:112-21. https://doi.org/10.1016/j.atherosclerosis.2020.12.005. 
45. Hou L, Xu M, Yu Y, Sun X, Liu X, Liu L, Li Y, Yuan T, Li W, Li H, Xue F. Exploring the causal pathway from ischemic stroke to atrial fibrillation: a network Mendelian randomization study. Mol Med. 2020;26(1):7. https://doi.org/10.1186/s10020-019-0133-y.

\section{Figures}

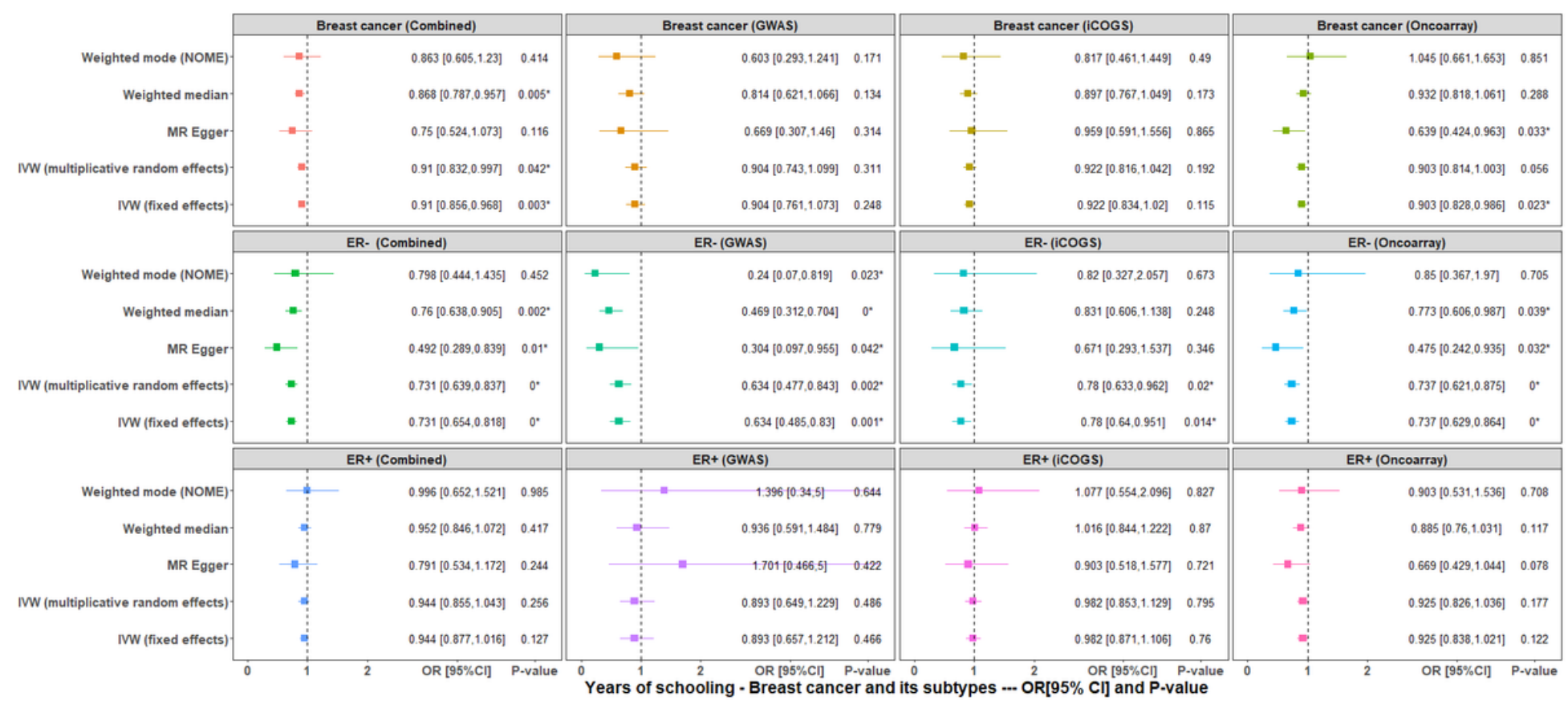

\section{Figure 1}

Causal effects of level of education on breast cancer and estrogen receptor subtypes. Breast cancer, overall breast cancer risk; ER+, estrogen receptor (ER)-positive breast cancer risk; ER-, ER-negative breast cancer risk. Four databases: (1) the OncoArray Consortium; (2) Collaborative Oncological Geneenvironment Study (iCOGS); (3) 11 other breast cancer genome-wide association studies (GWAS); (4) Combination of the above three databases. 


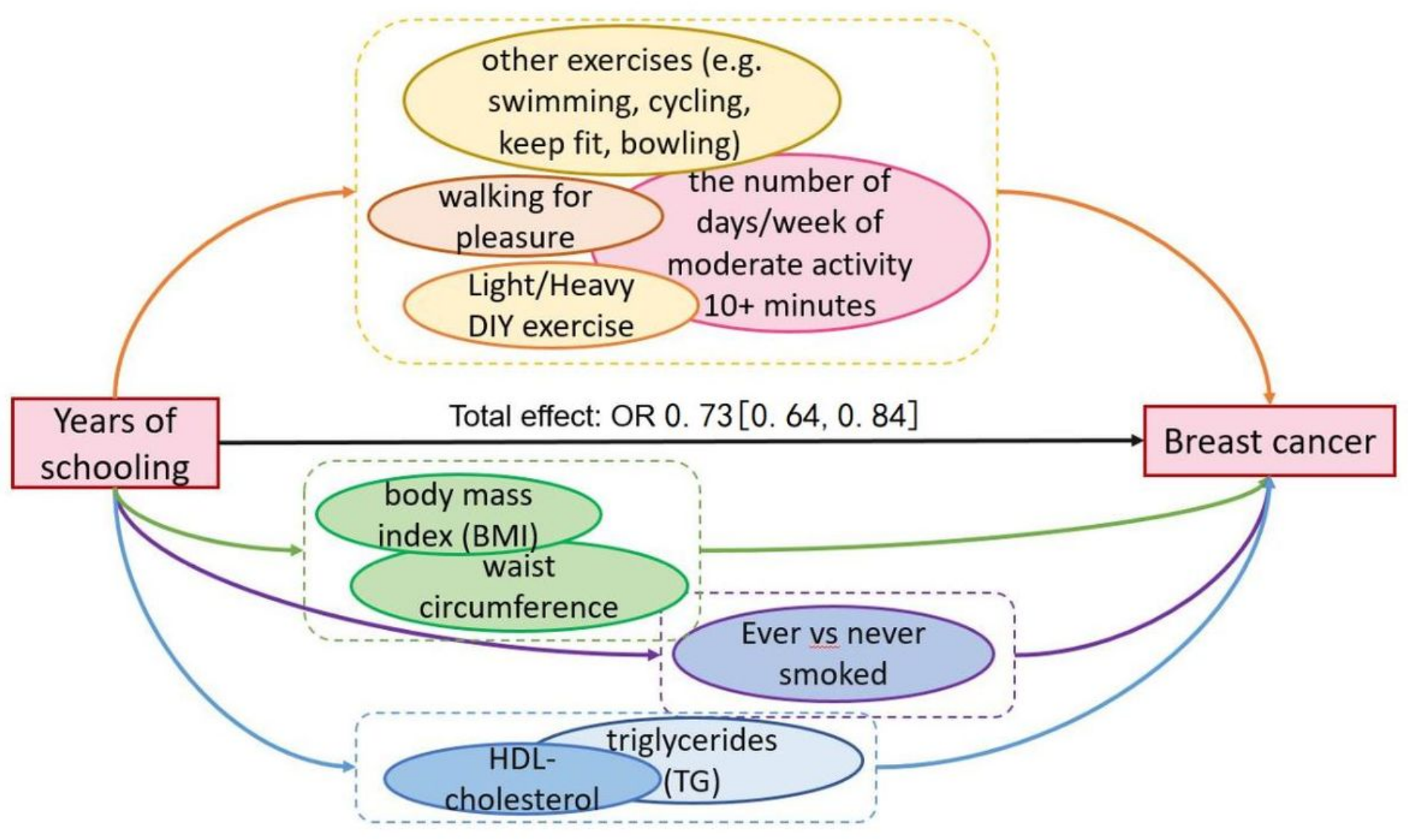

Figure 2

Diagram of causal pathway from years of schooling to breast cancer

\section{Supplementary Files}

This is a list of supplementary files associated with this preprint. Click to download.

- Additionalfile.pdf 